\title{
Identification of Deception Detection on Social Media (Twitter) Data Sets using Naive Base Classification and RVNN Model
}

\author{
N.Kanagavalli ${ }^{1}$, S.BaghavathiPriya ${ }^{2}$ and 3 S.Ilavarasan ${ }^{3}$ \\ \{kvalli.818@gmail.com ${ }^{1}$, baghavathipriya.s@ rajalakshmi.edu.in ${ }^{2}$, ilavarasan.sargunan@gmail.com $\left.{ }^{3}\right\}$ \\ Assistant Professor, Department of CSE, Rajalakshmi Institute of Technology, Chennai ${ }^{1}$, \\ Professor, Department of CSE, Rajalakshmi Engineering College, Chennai ${ }^{2}$, \\ Assistant Professor (SG), Department of IT, Saveetha Engineering College, Chennai ${ }^{3}$
}

\begin{abstract}
Twitter being a famous social media site not only helps people to share their thoughts in microblogs but also plays a pivotal role in situations of emergency for communication, announcement and so on. However, it results in anaversive effect when inappropriate tweet is reposted or shared to people thereby spreading rumors. This work describesthe methodologies in identifying the rumors using specific attributes like precision, fi-score, recall and support thereby solving the ranging rumor issues across the social media platform. A system detects candidate's rumor from twitter and then evaluates it applicably. The result of experiment shows the proposed algorithm in order to detect the rumors with acceptable accuracy.
\end{abstract}

Keywords: Rumor, Social media, CNN model, RvNN model,Twitter data set.

\section{Introduction}

This

The perils of rumor have an adverse consequence and it shall not be corroborated with any substantiation to prove its authenticity. Tweet means interact with massages to other users. Twitter having two users one is registered Users and another one is Unregisters Users. Registered users have all the rights like posts, tweet and retweet etc. Unregistered users have only read rights. The types of Misinformation are false news, reviews, listings, and more.Social psychology states that a rumor is allegedly claimed news without proper evidence thereby creating a chaos in the calm functioning world. As a matter of fact, it is hardly possible to envisage the trust worthiness of information circulated in social media. Demystifying the counterfeit information at the initial stage certainly paves way to limit the detrimental repercussions. The amalgamation of distrust information and deception developers' eventually impinges the regular flow of activities in the world of social media. The Existing websites which exclusively focuses rumor reporting in the social mediaare snopes.com and factcheck.org making effective strategies to detect the falseness of the information through manual verification process but fails to process the procedure with the aid of a proper coding system. The work attempts to bridge the gap between the conventional ideologies and to conceive a proper channel for better detection of the false information in the social media platform. 


\section{LITERATURE SURVEY}

This section embraces a review of the literature on various existing rumors detection on social media. These research papers are taken and reviewed according to the recent published years based on the rumors identification in social media.

\begin{tabular}{|c|c|c|c|}
\hline Authors & Methods & Advantages & Disadvantages \\
\hline $\begin{array}{l}\text { Jadhav S S and } \\
\text { Thepade S D [9] }\end{array}$ & $\begin{array}{lr}\text { Deep } & \text { Structured } \\
\text { Semantic } & \text { Model, } \\
\text { Recurrent } & \text { Neural } \\
\text { Networks } & \end{array}$ & $\begin{array}{l}\text { Detection accuracy is } \\
\text { high. }\end{array}$ & $\begin{array}{l}\text { Failed to integrate other social } \\
\text { media systems to extend this } \\
\text { method. }\end{array}$ \\
\hline Kaliyar R K et al. [2] & $\begin{array}{l}\text { A deep convolutional } \\
\text { neural network. }\end{array}$ & $\begin{array}{l}\text { Cross-entropy rate is less } \\
\text { in this method. }\end{array}$ & $\begin{array}{l}\text { User profile-based features } \\
\text { were not included for better } \\
\text { prediction of news articles. }\end{array}$ \\
\hline $\begin{array}{l}\text { Vishwakarma D K et } \\
\text { al. [3] }\end{array}$ & $\begin{array}{l}\text { Fake detection based } \\
\text { on maximally stable } \\
\text { extremal regions } \\
\text { detector. }\end{array}$ & $\begin{array}{l}\text { Better detection accuracy } \\
\text { was obtained. }\end{array}$ & $\begin{array}{l}\text { Not included splicing } \\
\text { localization algorithms for } \\
\text { image tampering. }\end{array}$ \\
\hline $\begin{array}{l}\text { de Oliveira N R et al. } \\
\text { [4] }\end{array}$ & $\begin{array}{l}\text { A computational } \\
\text { stylistic approach. }\end{array}$ & $\begin{array}{l}\text { Minimum overhead was } \\
\text { obtained in this method. }\end{array}$ & igh. \\
\hline Shu K et al. [5] & A principled method. & $\begin{array}{l}\text { Auxiliary information was } \\
\text { included to improve the } \\
\text { fake news detection. }\end{array}$ & $\begin{array}{l}\text { Failed to identify user profiles } \\
\text { for enhanced feature } \\
\text { extraction. }\end{array}$ \\
\hline Shu K et al. [6] & $\begin{array}{l}\text { A Data } \\
\text { method. }\end{array}$ & $\begin{array}{l}\text { Better classification } \\
\text { accuracy was achieved. }\end{array}$ & $\begin{array}{l}\text { Failed to include effective } \\
\text { visual features, such as } \\
\text { traditional local and global } \\
\text { features. }\end{array}$ \\
\hline Han Y et al. [7] & $\begin{array}{lr}\text { Graph } & \text { Neural } \\
\text { Networks } & \text { with } \\
\text { Continual } & \text { Learning } \\
\text { Method. } & \\
\end{array}$ & $\begin{array}{l}\text { Computational time was } \\
\text { very fast. }\end{array}$ & $\begin{array}{l}\text { The developed classifier is not } \\
\text { performed well on other } \\
\text { environments, like Gossip } \\
\text { Cop. }\end{array}$ \\
\hline Zhang J et al. [8] & $\begin{array}{l}\text { Deep Diffusive } \\
\text { Network Model. }\end{array}$ & $\begin{array}{l}\text { Detection accuracy was } \\
\text { high in this method. }\end{array}$ & $\begin{array}{l}\text { Computation time will be } \\
\text { extended } \\
\text { heterogeneous network }\end{array}$ \\
\hline
\end{tabular}




\section{HARDWARE \& SOFTWARE REQUIREMENTS:}

- This project is executed with the help of Deep Learning Python libraries. Anaconda Navigator, Pythonlibraries(Tensorflow, Keras)

\section{MODULE EXPLANATION:}

\section{MODULE 1 - (PRE-PROCESSING MODULE)}

Extracting the relevant fields from the dataset is the preprocessing step. The relevant fields include tweet ID, tweet reply status ID, tweet text, screen name. In which screen name is of little importance and the other three are used for further purposes. After extracting these fields from json, write them to csv files for the rest of the work.

MODULE 2 (POLARITY IDENTIFICATION MODULE)

In this module, each tweet's sentiment polarity will be analyzed. This polarity is also considered as one feature for CNN and RNN. Tweets were extracted from the tweets column from the csv file and the polarity is calculated using Sentiment Intensity Analyzer from the nltk sentiment vader. Each tweet is given as an input to the polarity score calculator and returns a dictionary with 4 values, negativity, positivity, neutral and compound.

Fig 1: DATAFLOW FOR RUMOUR DETECTION

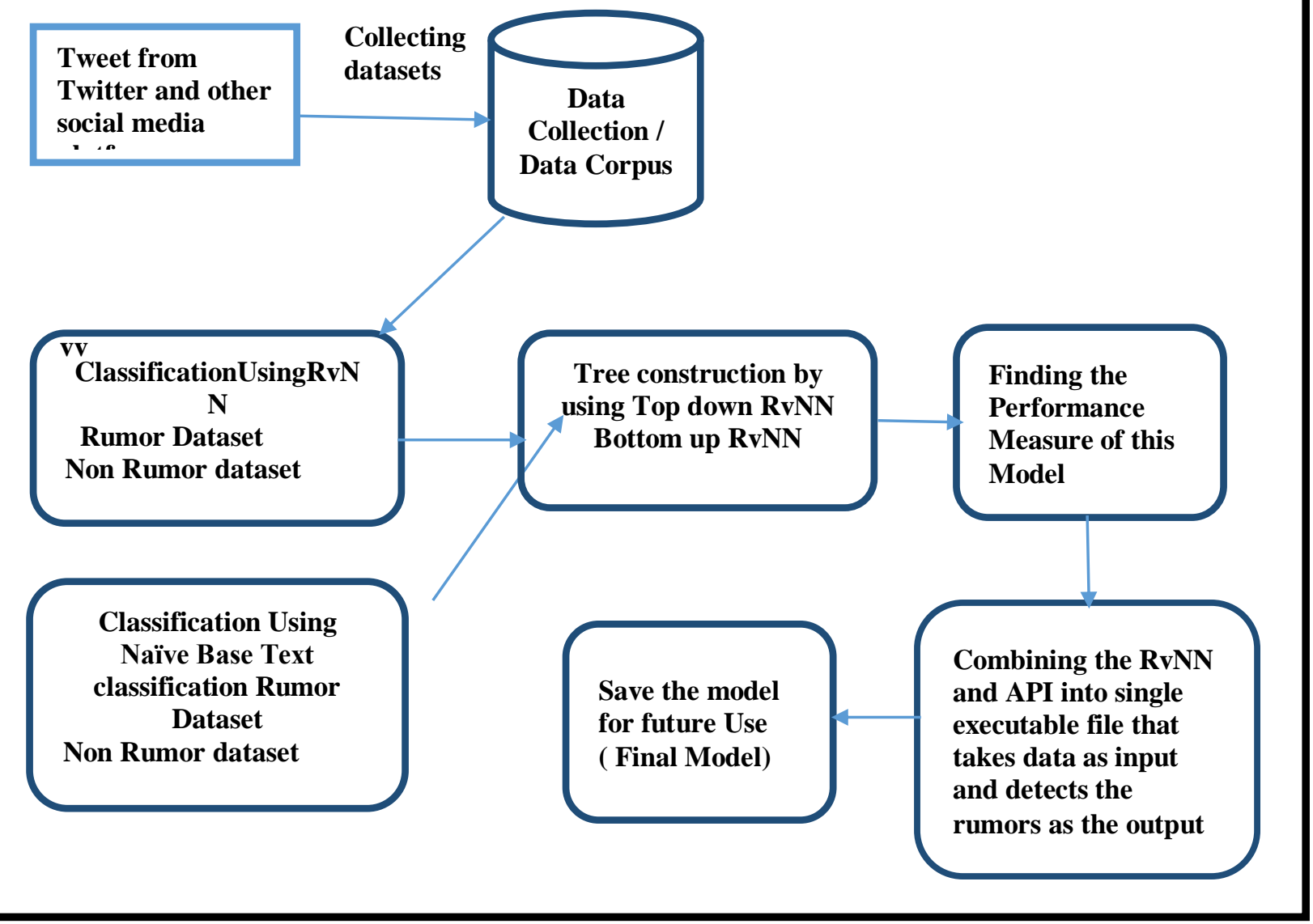




\section{MODULE 3 (TREE STRUCTURING MODULE)}

This module is one of the main tasks in the proposed model and this part provides structure for each csv file input. It is implemented by parsing through each file and identifying the parent node and child nodes based on ID and response tweet ID.The first figure is Top down RvNN Model, and the second figure is Bottom up RvNN Model.
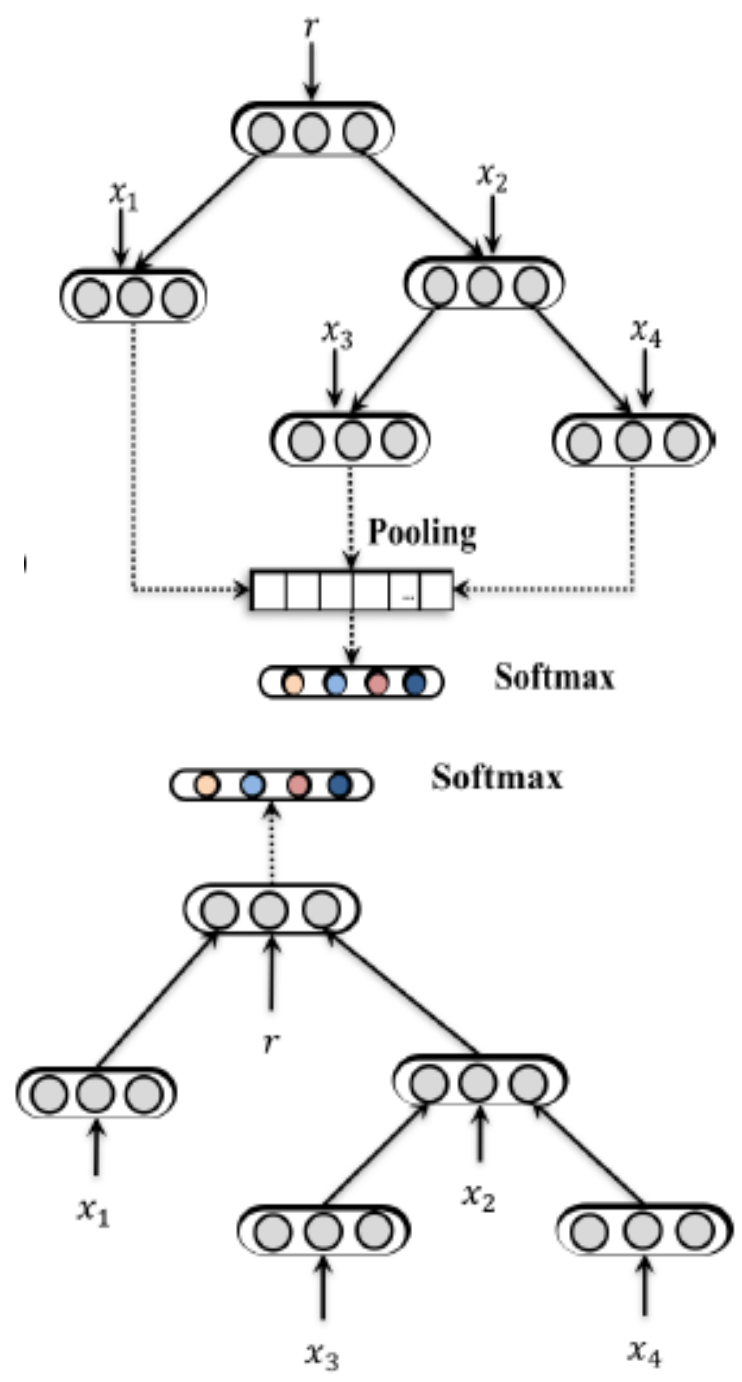

MODULE 4 (NEURAL NETWORK MODEL CREATION)

- In this module, 4types of neural network model is building using CNN, RNN, CNN $+\mathrm{RNN}$ and RvNN. First three models is based only on text and RvNN model is based on structure as well as content based. 


\section{METHODOLOGIES}

\section{NB Classification}

Naive base classification is the preeminent method for text classification. This is based on baye's theorem concept oriented model. It originates the probability of the given Item's vector are assigned with a specific label. Generally Naive Bayes is known asdirect and quick classification algorithm, which is appropriate for a hugeportion of data.There are totally 3 types of NB classifier. 1. Gaussian Naive Bayes classifier: Especially used for when items are in continuous. 2. Multinomial Naive Bayes Classifier: It is used when items follow a multinomial distribution. 3 Bernoulli Naive Bayes classifier: It is mainly used when items are in the type of Boolean.

For example the datasets are collected and completed as on 30/03/2021. The four datasets as an example data for this work.

The election was over on $1^{\text {ST }}$ April 2021 -False

Tamilnadu Election date is April $6^{\text {th }} 2021$ - True

Tamilnadu people waiting eagerly for the election - True

It was a close election - False

Find the statement "Tamilnadu election over on April 6th 2021" is True or false

STEPS INVOLVED IN NB CLASSIFICATION

Step1: Take the probability value for each and every item in the given statement then multiply and get the results.

Step2: $\mathrm{P}($ Tamilnadu $) * \mathrm{P}($ election $) * \mathrm{P}($ over $) * \mathrm{P}($ on $) * \mathrm{P}($ April $) * \mathrm{~A}(6$ th $) * \mathrm{P}(2021)$

Step3: Need to identify the two attributes such as True and false.

Step4: Total words in True list $=14$

Step5: Total words in False list $=13$

Step6: Total Unique words in list $=19$

\section{Step 7:}

Find 1. P(Tamilnadu election over on April 6th 2021/True)

$\mathrm{P}($ Tamilnadu/True $)=2 / 14$

$\mathrm{P}($ election/True $)=2 / 14$

$\mathrm{P}($ Over $/$ True $)=0 / 14$

$\mathrm{P}(\mathrm{On} /$ True $)=0 / 14$

$\mathrm{P}($ April/True $)=1 / 14$

$\mathrm{P}\left(6^{\text {th }} /\right.$ True $)=1 / 14$

$\mathrm{P}(2021 /$ true $)=1 / 14$

$\mathrm{P}($ Tamilnadu/True $) * \mathrm{P}($ election/True $) *$ $\mathrm{P}($ April/True $) * \mathrm{~A}(6$ th/True $) * \mathrm{P}(2021 /$ True $)$

$\mathrm{P}(\text { over/True })^{*} \quad \mathrm{P}(\text { on/True })^{*}$

Answer $=0$ 
To rectify the above results using Laplace smoothing formula, that is as follows

$\mathrm{P}$ (Choosen Word)

$=$

(Choosen count +1$)$

$\mathrm{P}($ Tamilnadu/True $)=3 / 33$

(total number of word + Number of unique words)

$\mathrm{P}($ election/True $)=3 / 33$

$\mathrm{P}($ Over/True $)=1 / 33$

$\mathrm{P}(\mathrm{On} /$ True $)=1 / 33$

$\mathrm{P}($ April/True $)=2 / 33$

$\mathrm{P}\left(6^{\text {th }} /\right.$ True $)=2 / 33$

$\mathrm{P}(2021 /$ true $)=2 / 33$

$\mathrm{P}($ Tamilnadu/True $) * \mathrm{P}(\text { election/True })^{*}$

$\mathrm{P}(\text { over/True })^{*} \quad \mathrm{P}(\text { on/True })^{*}$

$\mathrm{P}($ April/True $) * A(6$ th/True $) * \mathrm{P}(2021 /$ True $)$

Answer $=1.68940944 \mathrm{e}^{-9}$

Find 2. P(Tamilnadu election over on April 6th 2021/False)

$\mathrm{P}($ Tamilnadu/False $)=0 / 13$

$\mathrm{P}($ election/False) $2 / 13$

$\mathrm{P}($ Over/False $) 1 / 13$

$\mathrm{P}($ On/False $) 1 / 13$

$\mathrm{P}($ April/False $)=1 / 13$

$\mathrm{P}\left(6^{\text {th }} /\right.$ False $) 0 / 13$

$\mathrm{P}(2021 /$ False $) 1 / 13$

$\mathrm{P}($ Tamilnadu/False $) \quad \mathrm{P}($ election/False $) \quad * \mathrm{P}($ over/False $) \quad * \quad \mathrm{P}($ on/False $) *$ $\mathrm{P}($ April/False $) * \mathrm{~A}(6$ th/False $) * \mathrm{P}(2021 / \mathrm{False})$

Answer $=0$

To rectify the above results using the same above formula,

$\mathrm{P}($ Tamilnadu/False $)=1 / 32$

$\mathrm{P}($ election $/$ False $)=3 / 32$

$\mathrm{P}($ Over $/$ False $)=2 / 32$

$\mathrm{P}($ On/False $)=2 / 32$

$\mathrm{P}($ April $/$ False $)=2 / 32$

$\mathrm{P}\left(6^{\text {th }} /\right.$ False $)=1 / 32$

$\mathrm{P}(2021 /$ False $) 2 / 32$

$\mathrm{P}($ Tamilnadu/False $) \quad \mathrm{P}($ election/False $) \quad * \mathrm{P}($ over/False $) \quad * \quad \mathrm{P}($ on/False $) *$ $\mathrm{P}($ April/False $) * \mathrm{~A}(6$ th/False $) * \mathrm{P}(2021 /$ False $)$

Answer $=1.39698386 \mathrm{ee}-9$

Step 8: compare these 2 results. The first one is higher value compared to another one. 
Step 9: Higher value is depend on the True attributes

Step 10: The given statement "Tamilnadu election over on April 6th 2021" is comes under 'True"

\section{STEPS INVOLVED IN RVNN MODEL}

1. Install anaconda

2. Import python libraries

importnumpy as $\mathrm{np}$

import pandas as pd

importmatplotlib.pyplot as plt

3. Read two files one is original Information and another one is rumor information

4. Set label value as true $=0$ and false $=1$

5. Convert all the text files into numerical values

6. Combine both information into a single file using pandas function pd.concat ([dataset1, dataset2])

7. Check balanced or unbalanced dataset

8. Shuffle or Resample by using code

dataset $=$ dataset. $\operatorname{sample}($ frac $=1)$ dataset.head $(20)$

9. Import Natural Language Tool Kit packages(nltk)

10. from the nltk.corpus import stopwords

11. from the nltk.stem import WordNetLemmatizer

12. Data cleaning by using the function defcleaning_data(row):

13. This function returns the cleaned information

14. Then perform feature extraction by importing tfidVectorizer

15. Model selection by using this code

fromsklearn.model_selection import train_test_split

train_data ,test_data, train_label , test_label $=$ train_test_split $(\mathrm{x}, \mathrm{y}$, test_size $=0.2$ ,random_state $=0$ )

16. Perform vectorization for train data and test data

vec_train_data $=$ vectorizer.fit_transform(train_data)

17. Check the balanced partition using this line of code

train_label.value_counts()

test_label.value_counts()

18. Develop model by using Naïve Base

fromsklearn.naive_bayes import MultinomialNB

fromsklearn.metrics import accuracy_score,classification_report

19. Accuracy and precision and recall are calculated by using the formula. The result is as follows 


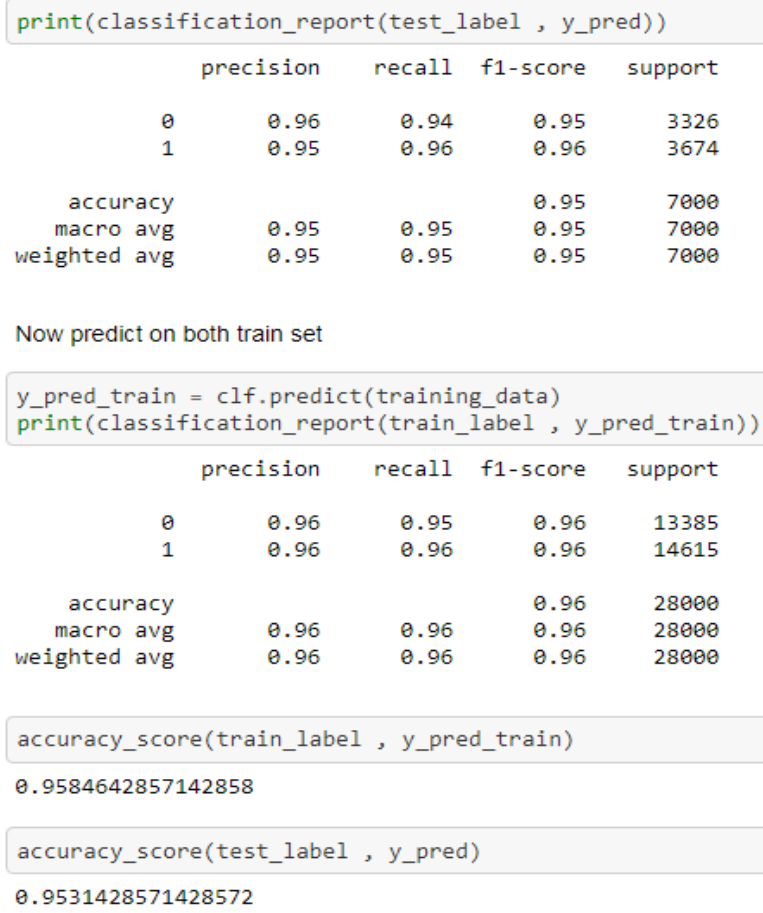

20. Finally save this model using joblib library file.

\section{RESULTS}

Calculate the given information is original or rumor from the trained data model. If the output of the array shows the numerical value 1 then the information is rumor. Or else the output of the array shows the numerical value 0 then the information is Original information.

\section{Output}

info= cleaning_data(str(" Tamilnadu Election will get over by April 1 2021."))

single_prediction $=$ clf.predict $($ vectorizer.transform $([$ info] $]$.toarray ()$)$

single_prediction

\section{Output}

$\operatorname{array}([1])$

This result shows that the given information is Rumor.

info= cleaning_data(str(" Tamilnadu Election will get over by April 6 2021."))

single_prediction $=$ clf.predict $($ vectorizer.transform $([$ info] $]$.toarray ()$)$

single_prediction

Output array([0])This result shows that the given information is Original Information 


\section{Conclusion}

Most people are gathering news online today. Everyone is free to get and share information on social networks anywhere at any time. So, in social media, breaking news spread very quickly. Sometimes rumors spread rapidly in social media with breaking news, which can also harm society and government.In that need to take some steps to prevent this rumor from spreading. There are different rumour detection techniques that use machine learning, deep neural network, etc. This work focuses primarily on a comparative study of different neural networks for social media rumour detection. Lots of work is done in the detection of rumors using neural networks that make use of CNN and RvNN.

\section{References}

[1] Ozbay F A and Alatas B, "Fake news detection within online social media using supervised artificial intelligence algorithms", Physica A: Statistical Mechanics and its Applications, vol.540, pp.123174, February 2020.

[2] Kaliyar R K, Goswami A, Narang P, Sinha S, "FNDNet-A deep convolutional neural network for fake news detection", Cognitive Systems Research, vol.61, pp.32-44, January 2020.

[3] Vishwakarma D K, Varshney D, Yadav A, "Detection and veracity analysis of fake news via scrapping and authenticating the web search", Cognitive Systems Research, vol.58, pp.217-29, December 2019.

[4] de Oliveira N R, de Medeiros D S, Mattos D M, "A Sensitive Stylistic Approach to Identify Fake News on Social Networking", IEEE Signal Processing Letters, July 2020.

[5] Shu K, Wang S, Liu H, "Understanding user profiles on social media for fake news detection", Multimedia Information Processing and Retrieval (MIPR) IEEE, pp.430-435, April 2018.

[6] Shu K, Sliva A, Wang S, Tang J, Liu H, "Fake news detection on social media: A data mining perspective", ACM SIGKDD explorations newsletter, vol.19, no.1, pp.22-36, September 2017.

[7] Han Y, Karunasekera S, Leckie C, "Graph Neural Networks with Continual Learning for Fake News Detection from Social Media”, arXiv preprint, pp.2007.03316, July 2020.

[8] Zhang J, Cui L, Fu Y, Gouza F B, "Fake news detection with deep diffusive network model", arXiv preprint, pp.1805.08751. 2018.

[9] Jadhav S S and Thepade S D, "Fake news identification and classification using DSSM and improved recurrent neural network classifier", Applied Artificial Intelligence, vol.33, no.12, pp.1058-68, October 2019.

[10] Afroz S, Brennan M, Greenstadt R, "Detecting hoaxes, frauds, and deception in writing style online", In proceedings of IEEE Symposium on Security and Privacy, pp.461-475, May 2012.

[11] Hardalov M, Koychev I, Nakov P, "In search of credible news", In proceedings of International Conference on Artificial Intelligence: Methodology, Systems, and Applications, pp.172-180, September 2016.

[12] Shu K, Wang S, Liu H, "Beyond news contents: The role of social context for fake news detection", In Proceedings of the Twelfth ACM International Conference on Web Search and Data Mining, pp.312-320, January 2019.

[13] Zhou X and Zafarani R, "Network-based fake news detection: A pattern-driven approach", ACM SIGKDD Explorations Newsletter, vol.21, no.2, pp.48-60, November 2019. 